\title{
Impact of cost and perceived quality on utilisation of primary health care services in Tanzania: rural-urban comparison
}

\author{
M.A. MUNGA \\ National Institute for Medical Research, P.O. Box 9653, Dar es Salaam, Tanzania
}

\begin{abstract}
A cross-sectional study was carried out to assess the impact of perceived quality and cost of utilisation of primary health care services, in relation to malaria, in rural and urban districts in Tanzania. This study intended to explore whether there are differences between rural and urban users in terms of their perceptions of quality of health services and how these perceptions affect household decisions in utilising health services. The findings showed that socio-economic variables such as gender, income, education, wealth and household size were important in determining users' decision making on the amount and appropriate time to seek care and also mitigates effectively on the extent to which cost and perception of quality of care affect rural and urban users of health care services. The majority of rural households spent more time at the facility while waiting to be attended than urban users of health services. Female-headed households were more likely to use health care services more frequently than male-headed households. It was also shown that urban households used health care more frequently than rural ones; and lack of money was not as important as perceived quality both in relation to frequency in using nearby health facilities, and to the delay in seeking care. In conclusion, the perceived quality of health care services is a strong determinant of health care utilisation and it has a differential impact on utilisation of health services.
\end{abstract}

\section{Introduction}

Research and experience shows that however the health system is organised, barriers to seeking health care exist. These barriers may be financial, cultural, and institutional. Financial barriers relate to the fact that people may need health services but they cannot afford the costs associated with its utilisation. Culturally, peoples' theory of causation of illness may in most cases be a barrier to seeking care, especially in those societies with low levels of literacy. Moreover, health care institutions may shape peoples' perception of quality to the extent that potential users may opt not to use when they perceive it to be of poor quality. It is conventionally accepted that utilisation of health services is a complex phenomenon, which is determined by a complex set of factors.

It has been demonstrated in Burkina Faso that patients' perceptions of quality of care are critical to understanding the relationship between quality of care and utilisation of health services and that perceived quality of health services has a strong impact on utilisation patterns (Baltussen et al., 2002). The observed quality of care focuses merely on structural and process measures and relates to professionally defined standards of care and refers to whether health care services adhere to these standards (Donabedian, 1988).

Both in empirical and theoretical literature, cost of care has extensively been documented to be an important barrier to accessing and utilising health care. According to Hjortsberg and Mwikisa (2002), cost of access creates an especially problematic barrier to the poor people who need to seek care. It is further argued that in developing countries where distances to health facilities can be large, the infrastructures lacking and large number of people living below the poverty line, cost of access is a critical determinant of whether care is sought or not. It is worth noting that the cost of access is not limited to geographical accessibility alone, but also to things like consultation fees, opportunity cost of time lost for waiting at the facility as well as costs of drugs.

Cost of health care on the other hand is as important as user's perception of quality in determining consumer's utilisation of health care services. Typically, costs of health care services may be divided into two broad categories, namely direct and indirect costs. Direct costs relate to expenditures incurred by households in seeking care for the sick member. They include out of pocket expenditures for treatment, fees, drugs and cost of subsistence at a distant treatment site. Indirect costs on the other hand, relates to time costs of sickness/opportunity costs of the wages foregone by a sick person due to sickness. Generally these include travel and waiting time cost of caretakers, opportunity cost of healthy household members, time spent on treating or tending the sick or accompanying a sick person to the place of treatment.

Although there are many dimensions to notions of quality of health care, evidence shows that patients in sub-Saharan Africa consider the availability of drugs as one of the key indicators of health services (Leighton, 1995). It has been observed that patients 
are willing to bear the costs of drugs if they are assured that drugs will be available at the point of service (Litvak and Bodart, 1993). These observations are also supported by McPake et al. (1993) who pointed out that perceived quality is the most important factor as it influences the willingness of the population to pay for health services. Perceived poor quality of care has been seen to be one of the important barriers to seeking care (Becker et al., 1993). Various indicators of poor quality have been used to describe quality of care. For example, whereas Khan (1985) had used inadequacies in drug provision, Becker et al. (1993) and Key (1987) have used staff attitude and interpersonal relations between patients and health workers to conclude on quality of services.

The utilisation of primary health care services is usually a function of costs and consumers perceptions of quality of services. Utilisation would increase with increasing quality and vice versa. Utilisation would usually decrease if the costs of health care are unaffordable by consumers. Such information is not readily available for many districts of Tanzania. The aim of this study was to explore and describe the importance of costs and perceived quality of care on utilisation of primary health care services in the country. It also aimed at sorting out whether there are differences between rural and urban households in terms of how costs and their perception of quality affect their decision to utilise health care.

\section{Materials and Methods}

\section{Study site}

Two urban (Ilala and Temeke) and two rural (Kibaha and Bagamoyo) districts in eastern Tanzania were selected. The rural- urban selection was based on the assumption that there may be differences in terms of how the users in these two geographical areas are behaving in seeking health care both in response to their perceived quality, and/or costs of health care. The study employed both quantitative and qualitative data collection techniques.

This was a cross-sectional analytical study using data that was collected over the period between December 2002 and February 2003. A total of 826 households were selected using Epi-Info $(95 \%$ confidence interval) and divided into rural (50\% of the sample) and urban (50\% of the sample). Since certain parts of the study were primarily in rural/urban or semi rural/semi urban, the Ward boundaries were used to identify and locate appropriate study areas. This was done in respect of the fact that the technique is efficient, and it was hoped that the sample would be representative.

\section{Data collection techniques}

Semi- structured interviews with heads of households were conducted. The heads of households were enquired about inter alia, their experiences of health service utilisation during the last malaria illness episode and their general perceptions of quality of services in the area.

A documentary review was carried out to collect data on utilisation rates, drug stock-outs and number of staff at each health facility. As a result of poor record keeping, the information collected could not permit any logical and meaningful comparative analysis. To this effect, the study relied much on household survey data. In an attempt to enrich the researchers' knowledge on the subject matter, some important documents from the offices of District Medical Officers (DMOs) and Ministry of Health were consulted. Relevant studies, both published and unpublished were also reviewed.

\section{Data analysis}

Data was entered into Epi-Info database, consistency and amplitude errors were searched for and corrected and analysed using STATA 6.0. Both descriptive and regression analyses were performed so as to be able to assess the impact of selected variables on the utilisation of primary health care. Included in the ordinal probability logistic regression analysis were three variables namely education levels of the household heads, gender and household size.

\section{Results}

In this study respondents were categorised into two absolute monthly income groups, namely those who earned TShs. 30,000/- (US\$ 30) and above and those who earned below this threshold. The majority (57\%) of urban respondents reported to be earning an income of TShs 30,000/- or more as compared to $48 \%$ of rural respondents. The urban households were more likely to own more electrical goods than rural people. For instance, only $8.5 \%$ of rural households reported to own a video or television whereas $35 \%$ of respondents in the urban households reported to own these items at the time of interview. Interestingly, it was observed that $22.6 \%$ of respondents in rural households owned refrigerators as compared to $19.3 \%$ in urban households. 
Self-employment was the most common occupation among the rural (34.9\%) and urban (36.3\%) populations. However, formal employment was more common in the urban $(23.7 \%)$ than in rural $(13.6 \%)$ areas. Most of the respondents in all the districts had primary education. However, a large number of respondents without formal education were found in the rural districts than in the urban districts (Table 1). Female headed households were more common in urban $(31.2 \% ; \mathrm{N}=826)$ than rural areas $(13.1 \%)$. The opted for government health facility services when attacked by malaria. The proportion of rural households delaying seeking malaria treatment was found to be higher than that of urban households. Though the difference between rural and urban in terms of delaying care was not significant $(\mathrm{P}>0.05)$ it was found that about $50 \%$ of rural households stayed for a week before seeking care as compared to only $14 \%$ in urban households (Table 2). Lack of money

Table 1: Comparison of education level of the respondents in rural and urban districts

\begin{tabular}{llll}
\hline Level of education & $\%$ respondents in rural & $\%$ respondents in urban & Total \\
\hline Primary & $61.50 \%$ & $54.72 \%$ & $58.11 \%$ \\
Secondary & $10.17 \%$ & $21.07 \%$ & $15.62 \%$ \\
Post-secondary & $5.33 \%$ & $13.08 \%$ & $9.20 \%$ \\
No formal education & $23.00 \%$ & $11.14 \%$ & $17.07 \%$ \\
Total & $100 \%$ & $100 \%$ & $100 \%$ \\
\hline
\end{tabular}

rural household headed by women had larger mean family size (4.1) than their urban counterparts (3.5). When utilisation of health services was looked at in terms of frequency with which a household contacts a nearby government health facility, it was observed that the majority $(34.7 \%)$ of urban respondents were more likely to seek services at a nearby health facility than rural households. The majority of rural households (33\%) seemed to be more likely to opt for no care than urban households $(6 \%)$.

The study showed that only a small proportion (24\%) of rural respondents reported to have experienced malaria episode 14 days prior to the date of interview as compared to urban respondents (36\%). In the rural and urban districts, $49.4 \%$ and $57.4 \%$ respectively, attributed to the delay in seeking malaria treatment $(\mathrm{P}<0.05)$. Fifty-five percent of rural households cited money to be a major problem as compared to only $33 \%$ of urban households. The majority of rural households spent much more time at the facility while waiting to be attended than urban users of health services. In terms of delaying seeking health care, the findings showed a more or less similar trend as those exhibited by frequency of using health care. It was observed that as household size increased frequency of utilising health care decreased. Female-headed households were more likely to use health care services more frequently than male-headed households.

Table 2: Proportions of households who reported to have delayed seeking treatment of malaria

\begin{tabular}{llll}
\hline Time from onset of illness to seeking care & Rural & Urban & Total \\
\hline Less than a day & $15.53 \%$ & $20.67 \%$ & $18.58 \%$ \\
1 to 3 days* & $34.95 \%$ & $65.33 \%$ & $52.96 \%$ \\
7 week $^{*}$ & $49.51 \%)$ & $14.00 \%$ & $28.46 \%$ \\
TOTAL & $100 \%$ & $100 \%$ & $100 \%$ \\
\hline
\end{tabular}

* represents "delaying care" 
The majority of urban respondents $(65 \%)$ were more responsive to quality of health care services than their rural counterparts (45\%). A test of association, Pearson-Chi squared-test showed a significant $(\mathrm{P}<0.05)$ association between rural and urban dwellers and how one's decision to delay seeking care in a nearby health facility was affected by quality concerns. In terms of frequency in using care, urban households used health care more frequently than rural ones; and lack of money was not as important as perceived quality both in relation to frequency in using nearby health facilities and to their delay in seeking health care. When sensitivity to quality of care was compared it was found that $71.6 \%$ and $28.5 \%$ of the rural and urban populations, respectively was due to lack of funds. The difference was significant at $\mathrm{P}<0.05\left(\chi^{2}=\right.$ 14.5957). However, lack of trust in quality was associated with $48.4 \%$ and $51.6 \%$ of rural and urban populations, respectively.

The study findings indicated that perceived quality of health care services was a strong determinant of health care utilisation and it had a differential impact on utilisation of the services when rural and urban users were compared. There was a significant difference $(\mathrm{P}<0.05)$ on how quality of care was perceived between rural and urban users. Poor quality service was cited to be a reason for a delay in seeking care from health facilities among $47 \%$ and $71 \%$ of rural and urban households, respectively. Cleanliness of the premises, availability of drugs and staff were ranked as "fair" by the majority of the respondents in both rural and urban districts (Figure 2).

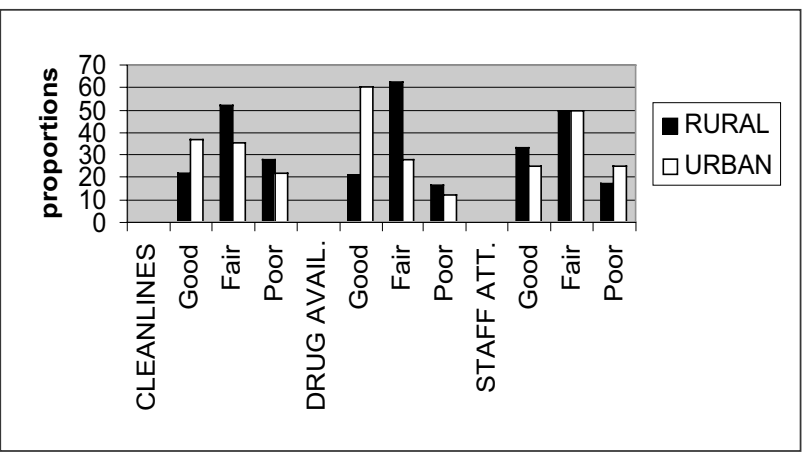

Figure 1: Proportions of respondents and their perceptions of quality of health services

Waiting time at health facilities was observed to be an important factor influencing delay in seeking care. The majority of rural households spent more time at the facility while waiting to be attended than urban users of health services (Figure 2). However, there was no significant difference $(\mathrm{P}>0.05)$ in relation to the influence of waiting time on differential utilisation between rural and urban users. More than $50 \%$ of urban users had spent an hour or more at the government health facility waiting to be attended during the last malaria episode as compared to about $40 \%$ in rural households.

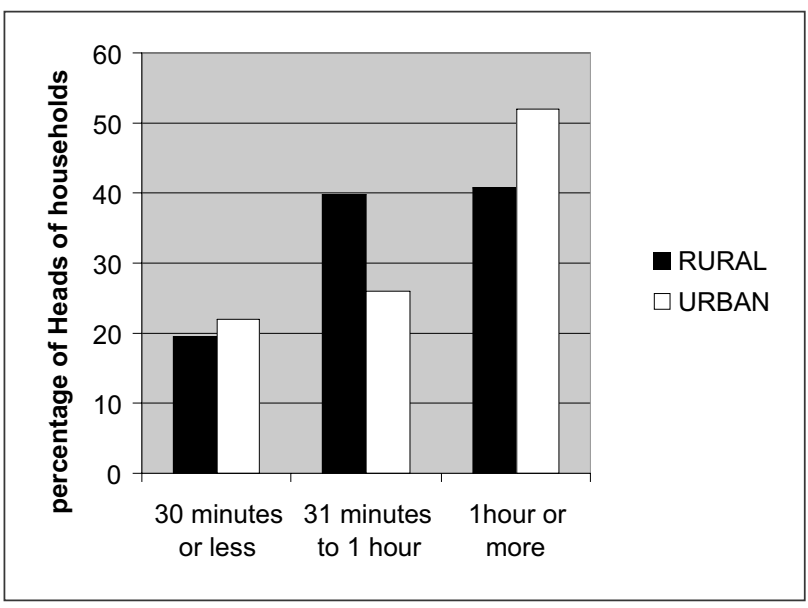

Figure 2. Time spent at the government facility waiting to be attended by the health worker

Self-medication was frequently practiced among urban than rural populations. On the other hand, more people in the rural communities consulted traditional healers than in the urban communities (Figure 3).

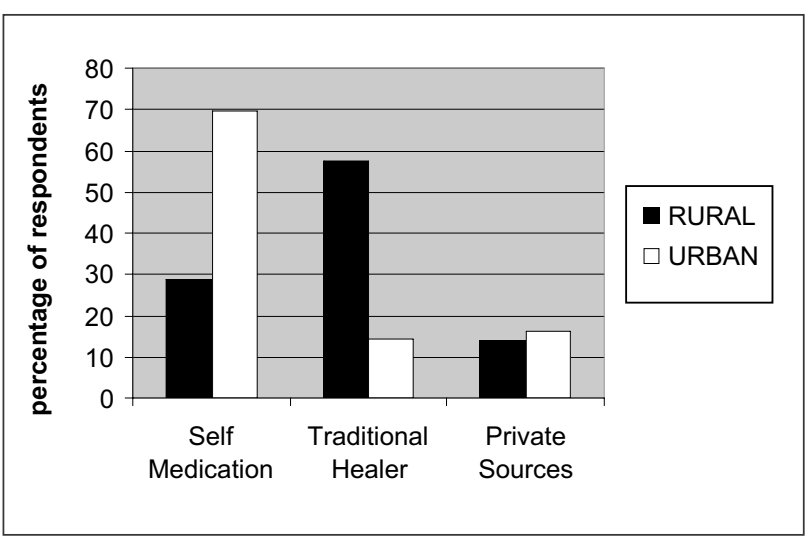

Figure 3. Sources of malaria treatment among non-users of government facilities

\section{Discussion}

The study findings highlight that, perceived quality of health care services is a strong determinant of health care utilisation and it has a differential impact on utilisation of health services. The findings indicate that 
perceived quality of care may increase demand for and eventual utilisation of health care services. It was shown in this study that urban households use health care more frequently than rural ones; and lack of money is not as important as perceived quality both in relation to frequency in using nearby health facilities, and to the delay in seeking care. This may probably be explained by not only low levels of education that may hamper rural peoples access to information necessary to assess the quality of services, but also different malaria morbidity patterns between rural and urban areas (data not shown).

Low level of education, income and little ownership of consumables indicate socio-economic inferiority among the rural households. Because of these factors, it may be argued that the observed underutilisation of primary health care services among the rural households is due to their inability to meet both official costs such as consultation fees and unofficial costs such as bribes, which was cited to be some of the reasons for not seeking care as there are differential treatments among health services' users with those who can not afford unofficial fees been more disadvantaged.

Low education levels exhibited by the majority of rural heads of households may amount to lack of sufficient and necessary capacities required to provide an appropriate definition of quality of health care and may even contribute to failure of households to effectively evaluate the severity of illness and when to contact a health worker for prompt treatment. Most surveys in developing countries (Leighton, 1995, Litvack and Bodart 1993, Becker et al., 1993) have shown that rural peoples' lack of capacity to pay for inter alia medicines is one of the major reasons for delaying and or not seeking treatment. Such observations are supported by the findings of this study. Furthermore, this study demonstrates that waiting time at the facility has an important bearing on differentiated utilisation of health care between rural and urban households. This is in consistence with the findings by Aday and Anderson (1984). The authors have shown that the opportunity costs of time is important in affecting people's decision making in utilising health care.

The findings of this study demonstrate that the majority of rural households spend much more time at the facility while waiting to be attended than urban users of health services. The long waiting time is likely to discourage users of health services in the rural areas to the extent that the community may be tempted to attribute waiting time to the observed health care utilisation differences between rural and urban users of health services. This shows that, though waiting time is not a significant determinant of health care utilisation it may partially explain the observed divergent utilisation trends between rural and urban users. The opportunity cost of time lost when patients wait for care at the facility and monetary costs such as those related to consultation fees and purchasing of drugs affect utilisation of services much more for rural than urban households. It can be deduced from this observation that if cost is a barrier to primary health care use, one direct effect is that the cost effectiveness of interventions will be reduced and the costs of malaria intervention will in the long run increase. According to Hjortsberg and Mwikisa (2002), reduced effectiveness of health care interventions is attributed to among other things, existing barriers that prevent consumers from using health care.

These observations further imply that policies such as user fees and subsidies or exemptions may have a much greater impact on utilisation of primary health care among users in rural than in urban communities. This is probably because rural communities are more responsive to cost of health care than the urban ones. The observations that the urban communities are more socio-economically better off than the rural counterpart have also been reported by others. Already, it has been documented that poor urban dwellers have better access and utilisation of health care than much of the rural population (WHO, 2000). This difference may be due to persistent low incomes of rural dwellers which limit their purchasing power of not only health services but also education which is very fundamental in imparting people's knowledge to effectively evaluate quality of care, and decide when it is appropriate to use it. Education level is known to have an impact on utilisation of care. With increased levels of education there has been observed possibilities that utilisation of services will go up. Education equips users with relevant tools to effectively evaluate the important aspects of quality and even can positively shape the users perception of severity of sickness and their perception of care. According to Becker (1965) household expenditure and consumption priorities may outweigh the importance of seeking care even when it is badly needed. In other words 'health care needs' and 'perceptions of severity' of sickness may be diverged in response to other 'perceived' important household expenditure and/or consumption needs, especially when heads of households are the sole providers of all day-to-day necessities. 
In terms of the impact of peoples' perception of quality on utilisation, the findings of this study provide basis to conclude that drug availability is an important predictor of quality of health care. It is reasonable to argue that maintaining a reliable supply of affordable drugs is critical to the success of the country's health policy in its effort to assure equity in utilisation of primary health care services. It has already been shown that people in developing countries tend to use health services as they believe that medical supplies such as drugs, will be available (Leighton 1995, Litvack and Bodart 1993).

In this study, utilisation of health care service was gender-related. Female-headed households are more likely to use health care services more frequently than male-headed households. This is probably because of the way much of African families are organised and may be because of the well known fact that women carry a heavy burden of poverty and ill health in almost all developing countries and they live in households where much of the decisions including those which affect their health are determined by conservative patriarchal governance structures. In most cases the women devote more time and attention to seek care for other members (for example, children) of the household.

Since utilisation of health services is affected by a complex set of factors (Becker et al., 1993) it is imperative for the ongoing health sector reforms to carefully address all the factors that may be a barrier to utilising health care with the same level of value as that attached to implementing the so called 'costeffective health interventions'. In other words it is important to strike a fair balance between the importance of equity and efficiency concerns in the design and implementation of health policies.

In conclusion, Tanzania seems to be increasingly segregated along socio-economic status of its people, with the important barriers to seeking health care services being quite different when low and high socio-economic households are compared. This study provides some evidence that perceived quality of care is an important determinant of utilization of care and it is more influential to urban than rural users. The consumers of health care in rural Tanzania are highly responsive to health care costs than they are to quality concerns.

\section{Acknowledgements}

I wish to thank the District Medical Officers for Kibaha, Bagamoyo, Ilala and Temeke for their assistance and enthusiasm. I am also grateful to my colleagues at the National Institute for Medical Research for their invaluable criticisms in the earlier version of the manuscript. Publication of this article is with the permission of the Director General, National Institute for Medical Research.

\section{References}

Aday, L.A. \& Anderson, R.M. (1984) Access to Medical Care in the U.S.: Who Has It, Who Doesn't. Pluribus Press, Inc., Chicago, IL.

Baltussen, R.M.P.M., Ye, Y., Haddad, S. \& Sauerborn, R.S. (2002) Perceived quality of care of primary health care services in Burkina Faso. Health Policy and Planning 17, 42-48.

Becker, G.S. (1965) A theory of the allocation of time. Economic Journal 299, 493-517.

Becker, S., Peters, D.H., Gray, R.H., Gultiano, C. \& Black, R.E. (1993) The determinants of use of maternal and child health services in Metro Cebu, The Philippines. Health Transition Review 3, 77-89.

Donabedian, V. (1988) The quality of care: how can it be assessed? Journal of the American Medical Association, 260, 1743-1748.

Hjortsberg, C.A. \& Mwikisa C.N. (2002) Cost of access to health services in Zambia. Health Policy and Planning 17, 71-72.

Key, P. (1987) Women, health and development, with special reference to Indian women. Health Policy and Planning 2, 58-60.

Khan, M.R. (1985) Evaluation of primary health care and family planning facilities and their limitations especially in the rural areas of Bangladesh. Dhaka, Bangladesh Institute of Development Studies.

Leighton, C. (1995) Overview: health-financing reforms in Africa. Health Policy and Planning 10, 213-222.

Litvack, J. \& Bodart, C. (1993) User fees plus quality equal improved access to health care: results of a field experiment in Cameroon. Social Science and Medicine 37, 369-381.

McPake, B., Hanson, K. \& Mills, A. (1993) Community financing of health care in Africa: an evaluation of the Bamako Initiative. Social Science and Medicine 36, 1383-1395.

WHO (2000) World Health Report, World Health Organization, Geneva. 